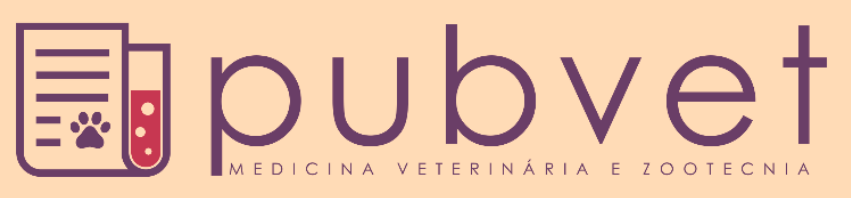

https://doi.org/10.31533/pubvet.v15n02a748.1-7

\title{
Osteossíntese de fratura de mandíbula consolidada
}

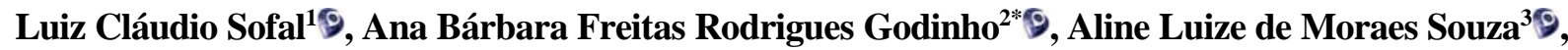 Isabel Cristina da Silveira Correa ${ }^{4} \mathbb{9}$}

${ }^{1}$ Médico Veterinário, Clínica Zoodonto. Serra-Belo Horizonte, Minas Gerais. Brasil.

${ }^{2}$ Professora da Universidade Estadual do Norte-Fluminense de Darcy Ribeiro, Departamento de Anatomia Animal. Rio de Janeiro, Brasil.

${ }^{3}$ Mestre em Ciência Animal - Universidade Estadual do Norte Fluminense de Darcy Ribeiro, Departamento de Anatomia Animal. Rio de Janeiro, Brasil ${ }^{4}$ MédicaVeterinária- Universidade Estadual do Norte Fluminense de Darcy Ribeiro, Departamento de Anatomia Animal. Campos dos Goytacazes -RJ Brasil.

*Autor para correspondência, E-mail: anaanatomiaanimal@gmail.com

Resumo. As fraturas mandibulares são comuns na clínica cirúrgica de pequenos animais e sua relevância está diretamente vinculada à importância funcional desse osso. Elas decorrem, normalmente, de traumatismos oriundos de atropelamentos, quedas e brigas, sendo a maior incidência entre o primeiro pré-molar e o segundo molar. $\mathrm{O}$ objetivo do tratamento de fraturas de mandíbulas será um retorno a uma função normal com uma oclusão dentaria perfeita ou satisfatória para que o animal possa voltar a se alimentar normalmente. Foi atendido na clínica veterinária Zoodonto, um paciente da espécie canina, fêmea, SRD, com três anos de idade, pesando $18,4 \mathrm{~kg}$, apresentando má oclusão dentária devido a fratura bilateral consolidada do corpo da mandíbula, entre o $1^{\mathrm{a}}$ e $2^{\mathrm{o}}$ molares inferiores direitos e entre o $1^{\circ}$ e $2^{\circ}$ pré-molares inferiores esquerdos. A técnica cirúrgica utilizada foi a fratura dos calos ósseos com cinzel e martelo, reposicionamento da mandíbula e fixação com fio de aço através da técnica não invasiva de amarria e resina acrílica bisacrilato. $\mathrm{O}$ conhecimento do tipo, frequência e terapias empregas em fraturas mandibulares é de fundamental importância para clínica veterinária. É necessário o aperfeiçoamento das técnicas de fixação, correção e estabilização de fraturas que ocorrem com maior incidência, aumentando assim a eficiência no seu tratamento e não somente o investimento em pesquisa de novos materiais e tecnologias que supram as limitações dos modelos terapêuticos. Neste sentido, este trabalho tem o objetivo de relatar um caso de fratura mandibular na qual a técnica de amarrilha culminou numa adequada estabilização e consolidação óssea.

Palavras-chave: Cão, consolidação, oclusão

\section{Osteosynthesis of consolidated jaw fracture}

Abstract. Mandibular fractures are common in small animal surgery and their relevance is directly linked to the functional importance of this bone. They usually result from injuries resulting from being run over, falling, and fighting, with the highest incidence between the first premolar and the second molar. The goal of treating jaw fractures will be to return to normal function with perfect or satisfactory dental occlusion so that the animal can return to eating normally. At the veterinary clinic Zoodonto, a canine species, female, SRD, with three years of age, weighing $18.4 \mathrm{~kg}$, presenting dental malocclusion due to consolidated bilateral fracture of the mandible body, between the 1st and 2nd lower molars between the 1 st and 2nd left lower premolars. The surgical technique used was a fracture of bone calluses with chisel and hammer, repositioning of the jaw, and fixation with steel wire using the non-invasive technique of bonding and acrylic resin bisacrylate. Knowledge of the type, frequency, and therapies used in mandibular fractures is of fundamental importance for a veterinary clinic. It is necessary to improve the techniques for fixing, correcting, and stabilizing fractures that occur with a greater incidence, thus increasing efficiency in their 
treatment and not only the investment in research of new materials and technologies that overcome the limitations of therapeutic models. In this sense, this work aims to report a case of mandibular fracture in which the technique of tying up culminated in an adequate bone stabilization and consolidation.

Keywords: Dog, consolidation, occlusion

\section{Osteosintesis quijada fractura de consolidado}

Resumen. Las fracturas mandibulares son comunes en la cirugía de animales pequeños y su relevancia está directamente relacionada con la importancia funcional de este hueso. Por lo general, resultan de lesiones causadas por atropellamientos, caídas y peleas, con la mayor incidencia entre el primer premolar y el segundo molar. El objetivo del tratamiento de las fracturas de la mandíbula será volver a la función normal con una oclusión dental perfecta o satisfactoria para que el animal pueda volver a comer normalmente. En la clínica veterinaria Zoodonto, una especie canina, hembra, mestiza, con tres años de edad, con un peso de $18.4 \mathrm{~kg}$, que presentaba maloclusión dental debido a una fractura bilateral consolidada del cuerpo de la mandíbula, entre el primer y el segundo molar inferior. entre el $1^{\circ}$ y $2^{\circ}$ premolares inferiores izquierdos. La técnica quirúrgica utilizada fue la fractura de callos óseos con cincel y martillo, reposicionamiento de la mandíbula y fijación con alambre de acero utilizando la técnica no invasiva de unión y resina acrílica bisacrilato. El conocimiento del tipo, la frecuencia y las terapias utilizadas en las fracturas mandibulares es de fundamental importancia para la clínica veterinaria. Es necesario mejorar las técnicas para reparar, corregir y estabilizar las fracturas que ocurren con mayor incidencia, aumentando así la eficiencia en su tratamiento y no solo la inversión en la investigación de nuevos materiales y tecnologías que superen las limitaciones de los modelos terapéuticos. En este sentido, este trabajo tiene como objetivo informar un caso de fractura mandibular en el que la técnica de amarrar culminó en una adecuada estabilización y consolidación ósea.

Palabras clave: Consolidación, oclusión, perro

\section{Introdução}

O termo sincrânio define o conjunto do neurocrânio, esplancnocrânio e da mandíbula, ossos que se articulam firmemente entre si e constituem a cabeça do animal. Dentre estes, a mandíbula é o único osso móvel e executa as funções básicas como mastigação, fonação e deglutição, além de participar da manutenção da oclusão dentária ocupando juntamente com a maxila a maior porção óssea do esqueleto facial (Busuito et al., 1986; Simões-Lopes, 2006; Smith \& Kern, 1995). O trauma de face pode acarretar lesões em estruturas ósseas do sincrânio, bem como em tecido mole facial (Rodrigues et al., 2018).

Não é possível se estabelecer um padrão terapêutico para as fraturas mandibulares. Cada caso deve ser avaliado detalhadamente e, portanto, fatores como o tipo de fratura, localização, raça, idade do animal, a presença ou ausência de dentes, e a oclusão dentária devem sempre ser considerados (Assunção, 2017). A presença dos dentes e de suas raízes, a necessidade de manutenção da oclusão e a baixa cobertura muscular devem ser considerados antes da escolha do melhor método (Legendre, 2005).

Existem vários métodos de tratamento para fraturas orais, podendo ser conservativos ou cirúrgicos. Os recursos como tomografia computadorizada, impressão 3D e os novos tipos de materiais biodegradáveis e mais resistentes são de extrema relevância no planejamento terapêutico individualizado, no entanto, alguns ainda são somente protótipos (Assunção, 2017).

A técnica da amarria interdental, utilizada nesse relato de caso, pode ser usada em fraturas graves. Os fios são colocados ao redor dos dentes para fixar os segmentos ósseos de forma parcial, colocandose sobre eles a resina acrílica. A remoção da resina deve ser feita de 4 a 6 semanas, com auxílio de fórceps odontológico universal (Gioso, 2007). A meta principal é o restabelecimento da oclusão normal e da função básica de mastigação, deglutição e oclusão dentária (Piermattei et al., 1997). 


\section{Material e métodos}

Foi atendido na clínica veterinária Zoodonto, em Belo Horizonte, Minas Gerais, um paciente da espécie canina, fêmea, SRD, com três anos de idade, pesando $18,4 \mathrm{~kg}$, apresentando sinais de má oclusão dentária (Figura 1).

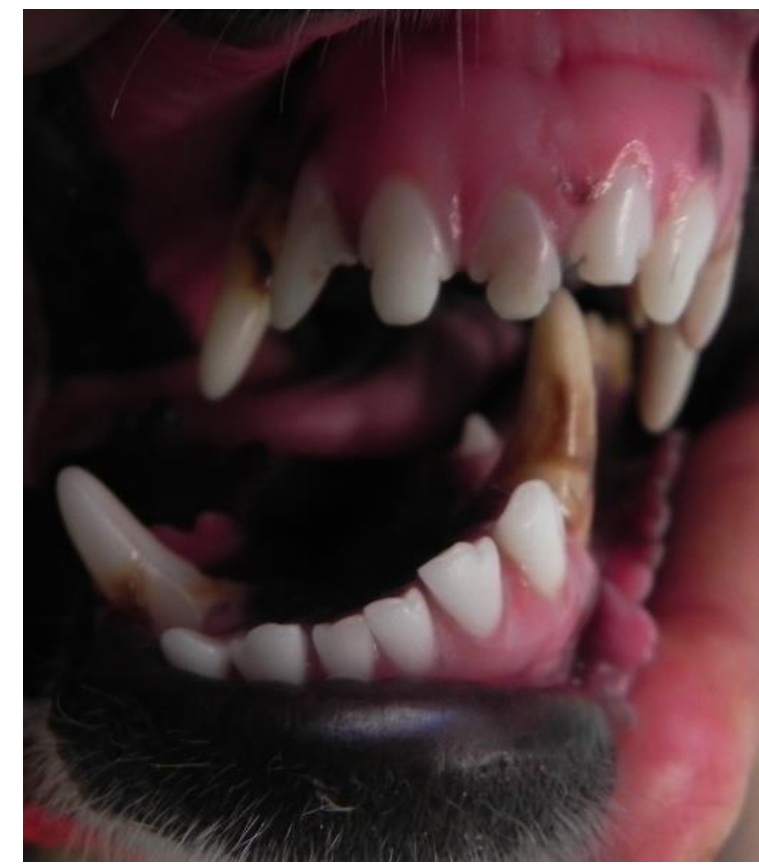

Figura 1. Vista rostral das arcadas dentárias de cão, evidenciando ausência total de oclusão dentária dos dentes anteriores.

Durante a anamnese e avaliação clínica do paciente, o tutor do animal relatou que havia encontrado o animal na rua, com suspeita de atropelamento. Após a realização de exames complementares foi possível confirmar, na avaliação radiográfica do sincrânio, a presença de fratura bilateral consolidada do corpo da mandíbula, entre o $1^{\mathrm{a}}$ e $2^{\circ}$ molares inferiores direitos e entre o $1^{\circ}$ e $2^{\circ}$ pré-molares inferiores esquerdos (Figuras 2A e 2B).
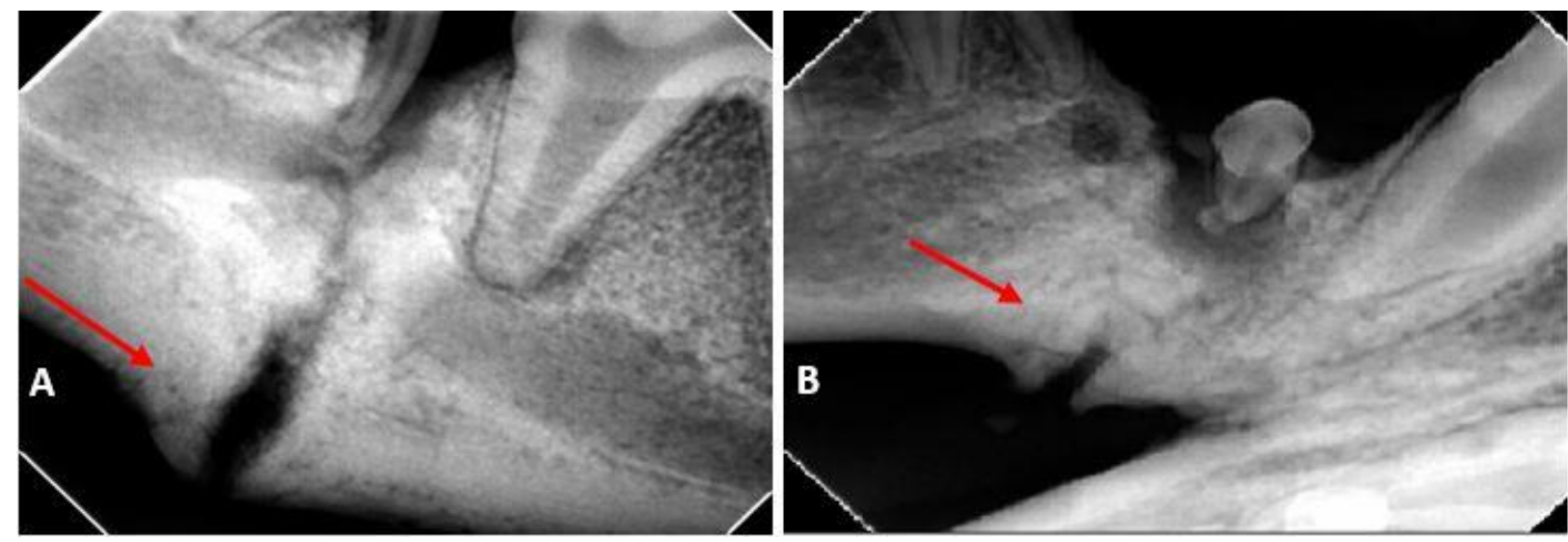

Figura 2. Imagens radiográfica dos corpos da mandíbula de cão. Fraturas consolidadas na hemimandibula direita, entre o $1^{\circ} \mathrm{e}$ $2^{\circ}$ molares inferiores (A) e na hemimadíbula esquerda entre o $1^{\circ}$ e $2^{\circ}$ pré-molares inferiores (B) (setas vermelhas).

O animal foi levado à sala de procedimentos odontológicos onde foi possível planejar a terapia cirúrgica para a correção mandibular. Inicialmente, foi projetado uma osteotomia, seguido de um realinhamento dos fragmentos numa relação anatômica satisfatória, por meio da aplicação de fios de aço inter dentários sustentado por resina acrílica. 
Como protocolo anestésico foi utilizado medicação pré-anestésica com acepromazina $1 \% 0,18 \mathrm{~mL}$ IM e meperidina $25 \mathrm{mg} / \mathrm{ml} \mathrm{0,7} \mathrm{mL} \mathrm{IM,} \mathrm{indução} \mathrm{com} \mathrm{propofol} \mathrm{0,9} \mathrm{mL} \mathrm{IV} \mathrm{e} \mathrm{manutenção} \mathrm{com} \mathrm{isoflurano}$ $1 \%$ e fentanil $1,8 \mathrm{~mL}$ IV. Com o animal em plano anestésico foi possível a observação detalhada das alterações relacionadas ao posicionamento normal dos dentes nas arcadas dentárias, em especial nas hemi-arcadas inferiores, direita e esquerda (Figuras 3A e 3B).

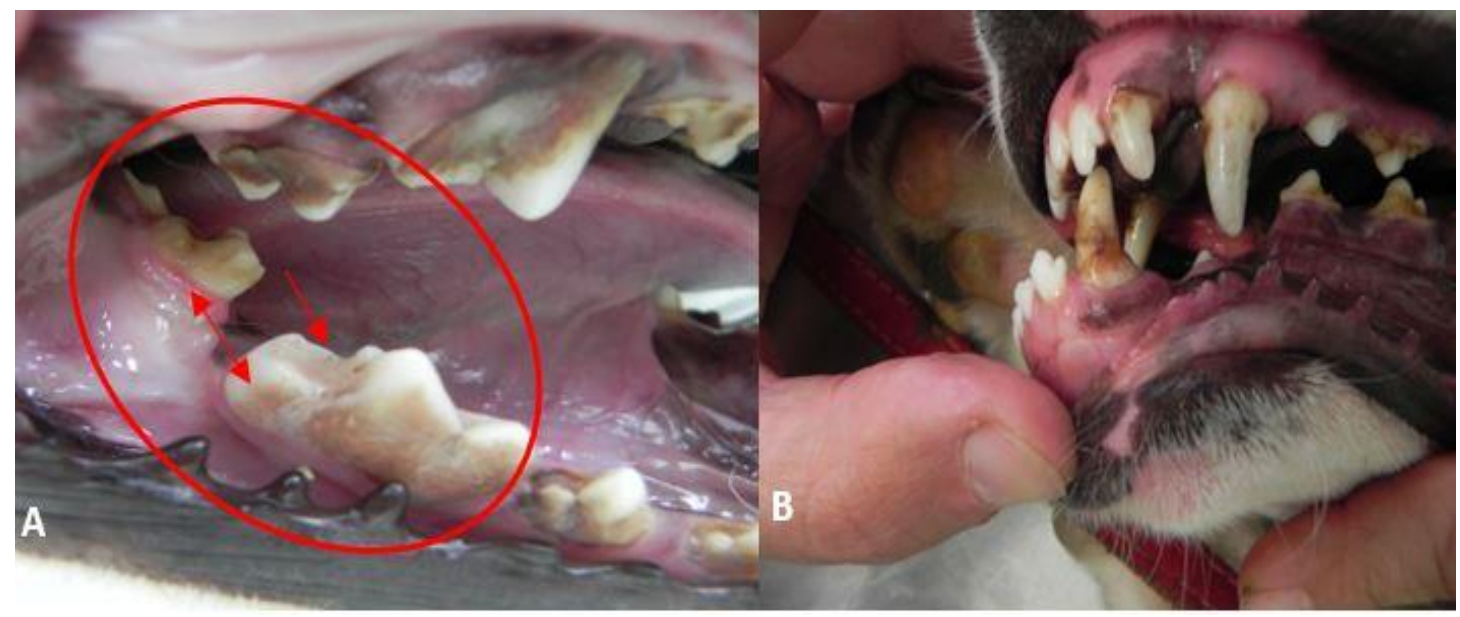

Figura 3. Faces vestibulares da dentição das arcadas superior e inferior de cão. Alteração na posição normal do $1^{\circ}$ e $2^{\circ}$ dentes molares da hemiarcada inferior direita $(\mathbf{A})$ e dos $1^{\circ}$ e $2^{\circ}$ pré-molares na arcada inferior esquerda $(\mathbf{B})$.

Para a realização da técnica cirúrgica o animal foi posicionado em decúbito dorsal e procedeu-se uma incisão na pele, na porção ventral do corpo da mandíbula. Após a exposição da referida estrutura foi realizada a fratura dos calos ósseos com cinzel e martelo e em seguida se deu o reposicionamento e fixação da mandíbula, utilizando fio monofilamentado de aço $0,20 \mathrm{~mm}$, com a técnica de amarria em todos os dentes da arcada inferior (Figura 4).

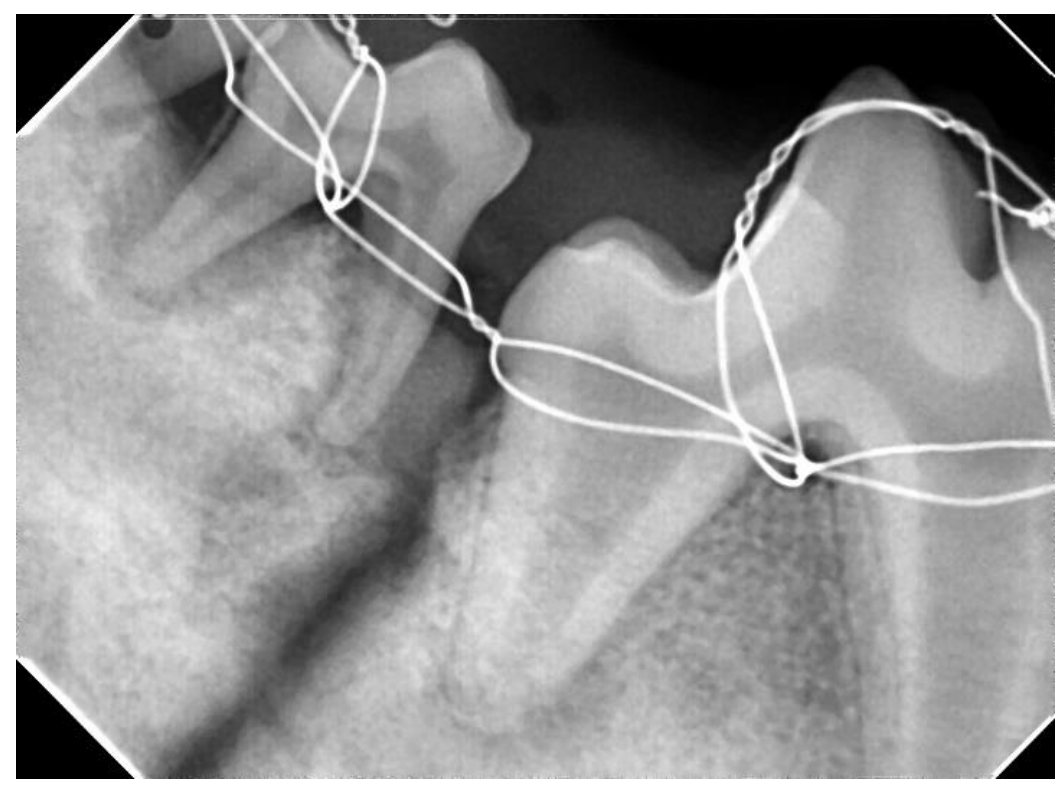

Figura 4. Imagem radiográfica da hemi-mandíbula direita evidencianado o reposicionamento dos dentes pré-molares $\left(1^{\circ} \mathrm{e} 2^{\circ}\right)$ após o emprego da técnica de amarria.

Após a colocação do fio, foi realizado o ataque ácido sobre o esmalte dos dentes ancorados com uso de ácido fosfórico, para promover melhor fixação da placa feita de resina acrílica bisacrilato que finalmente recobriu os dentes evitando deste modo o deslizamento do fio de aço. No pós-operatório foi realizada a antibioticoterapia - Stomorgyl 20, 1 comp. SID por 10 dias. 


\section{Resultados e discussão}

Apesar de existir uma maior prevalência na ocorrência das injúrias maxilares e mandibulares em cães com peso menor que $16 \mathrm{~kg}$, jovens e do sexo masculino (Kitshoff et al., 2013; Shamir et al., 2002), o caso em tela descreve um manejo cirúrgico de fratura de mandíbula para um animal de $18 \mathrm{~kg}$, três anos de idade, fêmea e que só foi assistido por um Médico Veterinário após a consolidação da referida fratura.

Desta forma, não foi possível determinar o escore de avaliação da fratura, (Freitas, 2017), apenas um grau acentuado de má oclusão, causada por uma consolidação incorreta da fratura. Os sinais e sintomas mais associados às fraturas de mandíbula são limitação da abertura bucal, edema, assimetria facial, mal oclusão, mobilidade atípica à manipulação, crepitação, parestesia e dor (Boffano et al., 2015; Gadicherla et al., 2016; Zarpellon et al., 2015).

A presença e a manutenção dos dentes são de grande importância para o sucesso da terapia de fratura mandibular (Fossum, 2018). Neste relato, a escolha pela técnica de amarria com fio metálico, associado ao uso de resina acrílica, tendo como suporte os dentes íntegros da arcada inferior se mostrou um modelo terapêutico bem-sucedido, gerando estabilidade e preservação da vascularização (Assunção, 2017). Uma avaliação previa e minuciosa foi realizada através das imagens radiográficas e a ausência de fragmentação e/ou perda óssea foram detalhes importantes para a aplicação de fio de aço interdental. Uma técnica considerada simples, efetiva e com poucos aspectos negativos relacionado (Harasen, 2008).

Após a correção cirúrgica, o animal passou a apresentar um grau satisfatório de oclusão dentária (Figura 5). Provavelmente, o reposicionamento dos dentes nas hemi-arcadas inferiores direita e esquerda o permitirá executar funções básicas como a mastigação e a deglutição (Simões-Lopes, 2006) (Figuras $6 \mathrm{~A}$ e B).

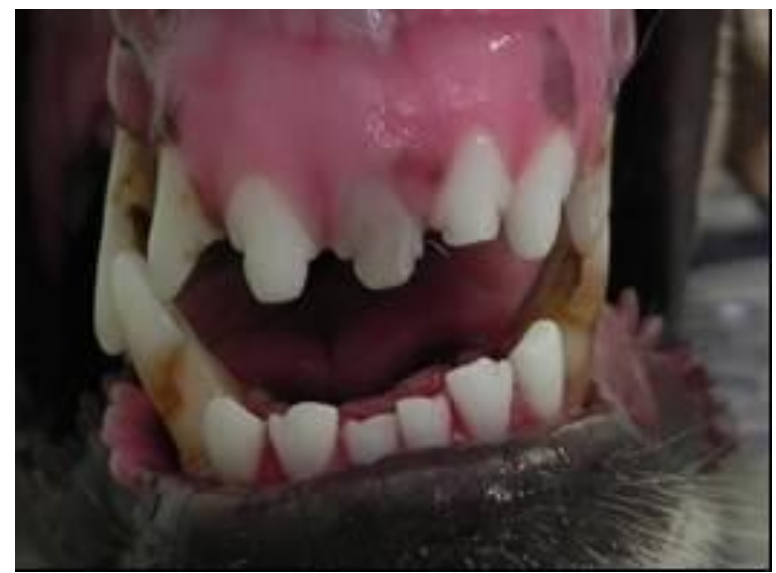

Figura 5. Faces vestibulares dos dentes incisivos superiores e inferiores de cão revelando grau de oclusão dentária satisfatório após procedimento cirúrgico corretivo.

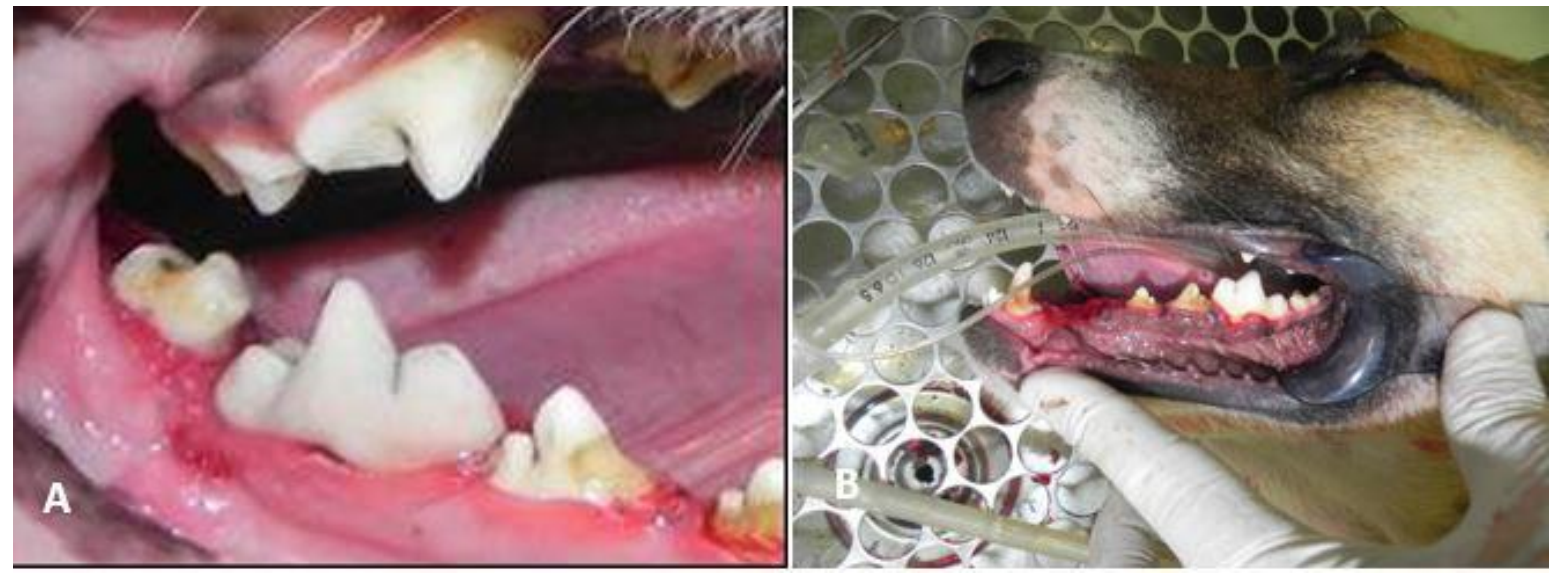

Figura 6. Faces vestibulares dos dentes molares na hemi-arcada inferior direita apresentando alinhamento e posicionamento normais (A). Faces vestibulares dos dentes pré-molares da hemi-arcada inferior esquerda demonstrando reposicionamento após procedimento cirúrgico corretivo $(\mathbf{B})$. 
A consolidação viciosa de fraturas de mandíbula ocorre em função de uma reparação óssea normal em cotos fragmentários posicionados anatomicamente de forma inadequada. Quando a consolidação viciosa está instalada, a osteotomia e o realinhamento dos fragmentos numa relação anatômica satisfatória, dependerá do comprometimento oclusal acarretado. Se esse for sutil, optasse por um tratamento conservador (movimento ortodôntico, desgaste oclusal, readaptação protética).

Em casos mais severos, preconiza-se a correção cirúrgica, em geral, através da osteotomia no traço de fratura (refratura) podendo estar associada à osteoplastia, a fim de proporcionar a correção funcional e estética (Jorge \& Gouveia, 1989).

A técnica de osteossíntese intra-oral com fio de aço associadas à resina acrílica instituída neste trabalho foi capaz de promover uma estabilização adequada após a redução das fraturas mandibulares identificadas. O êxito obtido com a utilização de fio monofilamentado de aço para estabilização do corpo mandibular se deu em função da técnica de amarria que permitiu uma fixação do mesmo desde o $3^{\circ}$ molar inferior direito até o $3^{\circ}$ molar inferior esquerdo passando pelos caninos e incisivos sob a forma de "oito". Com o intuito de evitar o deslizamento do fio de aço foi utilizada uma placa de resina acrílica

As miniplacas de titânio utilizadas em experimentos com cães e gatos promoveram uma fixação interna rígida, sendo de fácil manipulação e moldagem ao osso. Complicações em razão da sua aplicação podem ocorrer, como quebra dos implantes, assim como afrouxamento dos parafusos, ressaltando a necessidade de um manejo delicado dessas placas e desses parafusos para minimizar essas complicações (Gomes et al., 2010). No entanto, na medicina veterinária, poucos relatos descrevem a sua aplicação em cães e gatos (Bilgili \& Kurum, 2003; Boudrieau, 2004; Boudrieau \& Kudisch, 1996).

A escolha do método de estabilização nos casos de fraturas mandibulares depende particularmente de cada caso e do grau da fratura. Ao levarmos em consideração os fatores mecânicos, biológicos e clínicos em relação ao escore de avaliação da fratura (Freitas et al., 2013) ficou evidente que o tratamento de escolha, amarria interdental, associado à utilização de resina acrílica, foi o mais assertivo.

O temperamento dócil e tranquilo do animal e a capacidade do tutor em manter o animal em um semiconfinamento garantiram o sucesso da técnica cirúrgica. Além disso, medidas complementares como a oferta de uma alimentação pastoso, até uma completa osteosíntese, e aplicação de um colar Elisabethano foram essenciais para a evolução de sucesso do pós-operatório (Freitas et al., 2013).

Em fraturas mandibulares consolidadas, causando má oclusão, faz-se necessário a refratura dos calos ósseos e reposicionamento mandibular. No caso em questão, a técnica de osteossíntese não invasiva de amarria com fio de aço associada à resina acrílica se mostrou eficaz, promovendo estabilidade adequada no local da fratura. O baixo custo da técnica, a praticidade e a evolução positiva do pós-operatório tornam viável a indicação da referida técnica na rotina médica veterinária, melhorando o prognóstico e a sobrevida do animal. No pós-operatório foi alcançado uma oclusão satisfatória que permitiu ao paciente a realização de suas atividades funcionais.

\section{Referências}

Assunção, D. M. (2017). Técnicas terapêuticas de fratura mandibular em cães: revisão sistemática [Universidade Estadual Paulista (UNESP)]. http://hdl.handle.net/11449/156708

Bilgili, H., \& Kurum, B. (2003). Treatment of fractures of the mandible and maxilla by mini titanium plate fixation systems in dogs and cats. Australian Veterinary Journal, 81(11), 671-673. https://doi.org/10.1111/j.1751-0813.2003.tb12533.x

Boffano, P., Kommers, S. C., Karagozoglu, K. H., Gallesio, C., \& Forouzanfar, T. (2015). Mandibular trauma: a two-centre study. International Journal of Oral and Maxillofacial Surgery, 44(8), 9981004. https://doi.org/10.1016/j.ijom.2015.02.022

Boudrieau, R. J. (2004). Miniplate Reconstruction of Severely Comminuted Maxillary Fractures in Two Dogs. Veterinary Surgery, 33(2), 154-163. https://doi.org/10.1111/j.1532-950x.2004.04023.x

Boudrieau, R. J., \& Kudisch, M. (1996). Miniplate Fixation for Repair of Mandibular and Maxillary Fractures in 15 Dogs and 3 Cats. Veterinary Surgery, 25(4), 277-291. https://doi.org/10.1111/j.1532950X.1996.tb01414.x 
Busuito, M. J., Smith, D. J., \& Robson, M. C. (1986). Mandibular fractures in an urban trauma center. The Journal of Trauma, 26(9), 826-829. https://doi.org/10.1097/00005373-198609000-00008

Fossum, T. W. (2018). Small Animal Surgery E-Book (5th ed.). Elsevier Health Sciences.

Freitas, S. H., Dória, R. G. S., Minto, B.W., Nardi, A.B.,Melo, M. M., Camargo, L. M., Santos, M. D.,Shimano, A. C. \&Ambrósio, C, E. (2013). Haste intramedular modificada no tratamento de fratura diafisária de fêmur em cão - Relato de caso. Revista Brasileira de Medicina Veterinária, 35(4):323328.

Gadicherla, S., Sasikumar, P., Gill, S. S., Bhagania, M., Kamath, A. T., \& Pentapati, K. C. (2016). Mandibular Fractures and Associated Factors at a Tertiary Care Hospital. Archives of Trauma Research, 5(4). https://doi.org/10.5812/atr.30574

Gioso, M. A. (2007). Odontologia Veterinária: para o clínico de pequenos animais (2ed. Rev..). Minha Editora.

Gomes, C., Gouvêa, A. S., Alievi, M. M., Contesini, E. A., \& Pippi, N. L. (2010). Miniplacas de titânio na redução de fraturas mandibulares em cães e gatos: estudo de seis casos. Ciência Rural, 40(5), 1128-1133. https://doi.org/10.1590/S0103-84782010000500020

Harasen, G. (2008). Maxillary and mandibular fractures. The Canadian Veterinary Journal = La Revue Veterinaire Canadienne, 49(8), 819-820. https://pubmed.ncbi.nlm.nih.gov/18978981

Jorge, W. A., \& Gouveia, M. M. (1989). Correçäo cirúrgica de consolidaçäo viçosa de fratura de mandíbula. J. Health Sci. Inst, 19-23.

Kitshoff, A. M., Rooster, H., Ferreira, S. M., \& Steenkamp, G. (2013). A retrospective study of 109 dogs with mandibular fractures. Veterinary and Comparative Orthopaedics and Traumatology, $26(1), 1-5$.

Legendre, L. (2005). Maxillofacial fracture repairs. Veterinary Clinics of North America: Small Animal Practice, 35(4), 985-1008.

Piermattei, D. L., Flo, G. L., \& Camp, C. E. (1997). Fractures and luxations of the mandible and maxilla. In Handbook of small animal orthopedics and fracture repair (3.ed. Phil, Vol. 4, pp. 659-675).

Rodrigues, R. D., Quintas, P. H., Barreto, L. S., Costa, C. F. B., Silva, L. O. R., \& Aguiar, J. F. (2018). Manejo cirúrgico de fratura de mandíbula: relato de caso. Revista Da Faculdade de Odontologia $U P F, 23(3), 343-347$. https://doi.org/10.5335/rfo.v23i3.8643

Shamir, M. H., Leisner, S., Klement, E., Gonen, E., \& Johnston, D. E. (2002). Dog Bite Wounds in Dogs and Cats: a Retrospective Study of 196 Cases. Journal of Veterinary Medicine Series A, 49(2), 107-112. https://doi.org/10.1046/j.1439-0442.2002.jv416.x

Simões-Lopes, P. C. (2006). Morfologia do sincrânio do boto-cinza, Sotalia guianensis (P.J. van Bénéden) (Cetacea, Delphinidae). Revista Brasileira de Zoologia, 23(3), 652-660. https://doi.org/10.1590/S0101-81752006000300007

Smith, M. M., \& Kern, D. A. (1995). Skull Trauma and Mandibular Fractures. Veterinary Clinics of North America: Small Animal Practice, 25(5), 1127-1148. https://doi.org/10.1016/S01955616(95)50108-1

Zarpellon, A. T., Cruz, G. O. A., Gus, I. O., Moreira, G. H. G., Chamma, R. S., \& Masi, F. D. J. (2015). Review of 150 cases of mandible fracture between 2010 and 2013 at the Cajuru University Hospital, Curitiba, PR. Revista Brasileira de Cirurgia Plástica (RBCP) - Brazilian Journal of Plastic Sugery, 30(4). https://doi.org/10.5935/2177-1235.2015RBCP0200

Histórico:

Recebido: 1 de julho de 2020 .

Aprovado: 4 de agosto de 2020.
Licenciamento: Este artigo é publicado na modalidade Acesso Aberto sob a licença Creative Commons Atribuição 4.0 (CC-BY 4.0), a qual permite uso irrestrito, distribuição, reprodução em qualquer meio, desde que o autor e a fonte sejam devidamente creditados. 